Research Article

\title{
Interactive Marketing E-Commerce Recommendation System Driven by Big Data Technology
}

\author{
Yi Fu, ${ }^{1}$ Min Yang, ${ }^{1}$ and Di Han $\mathbb{D}^{2}$ \\ ${ }^{1}$ Chongqing City Vocational College, 402160 Yongchuan, Chongqing, China \\ ${ }^{2}$ ChongQing JianZhu College, Chongqing 400072, China
}

Correspondence should be addressed to Di Han; mandyfaye123@163.com

Received 18 September 2021; Revised 4 October 2021; Accepted 5 October 2021; Published 21 October 2021

Academic Editor: Tongguang Ni

Copyright (c) 2021 Yi Fu et al. This is an open access article distributed under the Creative Commons Attribution License, which permits unrestricted use, distribution, and reproduction in any medium, provided the original work is properly cited.

\begin{abstract}
This study combs through relevant literature, adopts a combination of typical sampling and random sampling, collects three big data technology-driven interactive marketing e-commerce companies in a specific period of Sina Weibo sample data for research, obtains historical information and data, and constructs a model. Through relevant analysis to eliminate invalid variables, we creatively selected three variables of Internet hot words, activities, and microtopics as independent variables and used marketing effects as dependent variables to carry out empirical analysis and study the marketing innovation of three representative companies based on big data technology. We discussed the use of self-media in interactive marketing e-commerce and the situation of marketing innovation based on self-media, focusing on the interactive relationship between marketing innovation and Internet word-of-mouth (brand image). Through research, we have derived the three-force model, which is the biggest result of this research, and provided a reference model for interactive marketing e-commerce companies to carry out follow-up marketing innovation based on the media. Limited to the level of research and ability, there are some deficiencies in the research, such as barrage marketing, big data marketing, and emotional computing, that have not been analyzed in depth. This article fully considers the dependence of small and medium e-commerce companies on e-commerce platforms in the era of big data and conducted detailed market research on their precision marketing strategies in the era of big data. This will be a new field that does not come from media marketing. This article intends to summarize a series of experiences and laws from special to general, from individuality to generality, so as to give full play to the role of personalized marketing in increasing website traffic and order conversion, in order to personalize the use of data by other e-commerce companies with marketing provides some valuable experiences and methods for reference.
\end{abstract}

\section{Introduction}

With the rapid development of information technology, the arrival of big data has brought new development opportunities for scientific research, business, and technological progress. Big data has become one of the important tools in the field of e-commerce [1]. In recent years, the country's e-commerce has ushered in a period of rapid development, and the transaction volume and scale of e-commerce have expanded rapidly [2]. On the other hand, the huge user and product base of e-commerce requires e-commerce companies to build precision marketing systems based on user needs and potential needs. The rapid development of information technology and the continuous progress of network technology have promoted the vigorous development of e-commerce. The development of e-commerce is profoundly changing people's consumption and living habits [3]. The total online retail sales have accounted for one-sixth of the total retail sales of consumer goods. E-commerce has become a powerful driving force that drives national consumption and promotes national economic growth. After nearly two decades of development, the country's e-commerce market has basically matured, and a domestic retail system represented by online retail giants, such as Taobao, JD.com, and Amazon, has formed, and the industrial competition pattern has basically taken shape [4-6].

Modern society is a society with rapid development of science and technology, and the ability to process 
information is continuously enhanced. Information is generated by human activities and fed back to people through data analysis techniques. Big data are the product born under this background [7]. The term big data has begun to be widely used by various media. Big data analysis methods are widely used in computer, cloud computing, Internet, and and other fields $[8,9]$. Scholars from all walks of life have carried out a lot of research on the methods of collecting, processing, analyzing, and using big data and achieved fruitful results [10]. Big data analysis methods take cloud computing technology as the main thrust and gradually penetrate into the analysis and research of various disciplines and industries. In addition, big data technology has been deeply integrated with enterprise management. At present, big data are not only used to promote enterprise management and market information collection but also combined with e-commerce has brought new development opportunities for all types of enterprises. The Internet and related industries have become the first source and demand side of big data [11]. E-commerce companies can use big data technology to analyze consumer needs through massive data mining, integrated analysis, and application, and they can provide consumers with personalized services and push notifications. With the continuous fermentation of big data technology, marketing strategies based on big data have also begun to be used by small- and medium-sized e-commerce companies, bringing many opportunities and challenges to e-commerce companies [12-14].

The purpose of this research is to integrate the relevant theories of the four disciplines of tourism, economics, marketing, and communication from a microperspective and examine the marketing innovation activities of interactive marketing e-commerce companies based on selfmedia. Therefore, the development of e-commerce big data research must be deeply integrated with relevant marketing strategies, which supported the basic strategies of precision marketing, combined with big data analysis technology, and explored the survival method of smalland medium-sized enterprises in the era of big data. Based on the gaps in academic research and the actual development needs of e-commerce companies, this article uses E-Commerce Co., Ltd. to study how e-commerce companies, especially small- and medium-sized e-commerce companies that rely on major e-commerce platforms, should adapt to the background of the era of big data. In the era of big data, we use big data technology to track changes in customer needs, better discover customer needs, cater to changes in customer needs, and carry out research on the development of enterprises. Through relevant research on the company's products, pricing, promotion, and business strategies, it discusses how smalland medium-sized e-commerce companies can do precision marketing from the perspective of the development and trend of small- and medium-sized e-commerce platforms. Through the introduction of statistical analysis, content analysis, and other techniques to establish a complete quantitative indicator system, we extract representative interactive marketing e-commerce companies, conduct comprehensive analysis and evaluation of interactive marketing e-commerce based on self-media marketing and marketing innovation, and analyze the actual situation. We stage interactive marketing e-commerce companies publish content and effects in operating Weibo accounts, sum up experience, and give suggestions for improving the marketing strategies of interactive marketing e-commerce companies.

\section{Related Work}

The research scope of foreign scholars is relatively wide. The first is the organization and operation of the tourism destination marketing system, marketing alliances, and their performance evaluation. The solution usually adopted abroad is to establish marketing alliances and conduct research on their development and operations. Secondly, the research also focuses on the characteristics, advantages, development issues, evaluation system, and operation of online marketing. In terms of operational research on tourism destination marketing systems, more emphasis is placed on the research on actual operational effects [15].

Malhotra and Rishi [16] referred to the tourism destination marketing system as a tourist information system. They believed that the basic theme and commonly used dominant function of DMS is to publish and process information and processing of all tourism activities-related organizations and attractions in a particular tourist area for reservation business and several core database-based application systems used in tourism information management, public information services, industry exchanges, and tourism online marketing. The e-commerce expansion model of the destination marketing system established by $\mathrm{Hu}$ [17] realized the three functions of commercial activities: online promotion, service provision, and transaction execution. From this perspective, the three functions of the destination marketing system are proposed with information and service provision functions, marketing functions, and transaction execution functions. Wright et al. [18] believed that the destination marketing system is an integrated marketing tool that gathers resources and services from a marketing perspective and is also a one-stop shopping center for tourists to choose and purchase products and services from the perspective of tourists. Elia et al. [19] research focused on how destination tourism information is delivered to tourists and how software and hardware equipment can meet tourists' needs. They gave particular attention to how national-level tourism agencies use this system to publicize and promote tourism destinations in the country. This concept is more appropriate to explain the functions of the national-level tourist agency information system, but its core focuses more on reflecting the function of information data processing, ignoring the other important functions of the system, namely, the interactive communication function and the needs of tourists for the function of the service. Liu et al. [20] pointed out that the destination marketing system should consider functions from two levels: management and information technology. At the management level, the system should achieve three major functions: information provision, marketing, and market research; at the technical level, 
three tasks must be completed: web design, website promotion, and website evaluation.

Other scholars believe that the tourism destination marketing system organically combines efficient Internetbased tourism promotion and marketing with local tourism consulting services to provide tourists with full service, which can greatly enhance the image of the tourist destination and the overall service level. The tourism destination marketing system is not only a destination information system but also a regional organization system. They pointed out that the tourism destination marketing system has competitive advantages in terms of network sales market agglomeration, the establishment of regional unified cognition, and the sharing of information resources [21]. Some scholars have introduced integrated marketing theories into the research of DMS, introduced the forms of integrating information resources, products, and services in the system, as well as introduced specific integrated methods for unified external publicity, promotion, and communication with customers. Scholars discussed it from multiple angles. Some experts also pointed out that marketing innovation is a new product, new process, or new system that has the potential to create a new market or change the existing market. They believe that marketing innovation is a general practice in a different industry, which is a jump in the rules of the game. In this way, the marketing process or the results of the marketing process are not the same as the conventional ones. Marketing innovation is a part of strategic innovation. It is a kind of corporate ability that can create value for customers, weaken competitors, and create wealth for stakeholders. The essence of innovation lies in the formation of a "communication element" and marketing activities that play its role so that users can have a sense of trust in their products and motivate them to buy. The communication element is expressed as an idea or concept, expressed as images, words, sounds, and symbols, which can give consumers a deep impression and redistribute. Researchers believe that there are two types of marketing innovation: incremental innovation and transformative innovation. Incremental marketing innovation is mainly product innovation, whereas transformative marketing innovation is technology oriented and market oriented $[22,23]$.

\section{Construction of an Interactive Marketing E-Commerce Recommendation System Driven by Big Data Technology}

3.1. Spatial Composition of Big Data Technology. Big data mean that it does not rely on sampling surveys but integrates all the data on a certain issue for analysis and processing. It is completely different from the method of sampling samples in terms of data sources and analysis methods. Big data contain the most complete reflection of the information of an event. Figure 1 shows a big data technology space architecture. Therefore, the information contained in big data is often far more than general data. The most complete information that can be reflected on the above. Compared with general data, big data contain a larger amount of information, which means more data.

The information contained in big data has gone beyond a single text form. The information of big data can be reflected in various forms, such as pictures, videos, audios, and geographic information, and it can also reflect a certain behavior from different dimensions. Therefore, the multidimensional nature of big data determines that an event can be reflected from more dimensions, and it also contains more useful information, but it also increases the difficulty of information processing.

$$
p(x(1), x(2), \ldots, x(n))=\prod_{i=1}^{n} p\{x(n) \mid x(n-i+1)\} .
$$

At present, there are many ways to apply big data at the technical and decision-making levels, and there is no fixed research method. Big data analysis technology is still in the integration stage.

$$
\begin{aligned}
C & =2 \times \frac{(p(x) \times p(n))^{1 / 2}}{(p(x)+p(n))}, \\
f(x) & =x(i)+\frac{x(i) \times x(j)}{x(i)+x(j)} .
\end{aligned}
$$

More importantly, big data are how $t_{o}$ effectively collect market data, and at the same time, on the basis of further processing and researching market data, it can obtain information that is beneficial to the organization or enterprise, obtain market dynamics, understand customer needs, and adjust it. The business strategy of an enterprise, obtaining greater profits, and the scope of market operations are all important content that big data can achieve for enterprise development.

$$
T^{2}-\frac{1}{n} \times \sum_{i=1}^{n}(p(i)-p(x))^{2}=0 .
$$

Because big data contain a large amount of information and reflect a large number of dimensions, and the factors that play a decisive role in a certain matter often only account for a small part, so in the processing of large-capacity but low-density information, data analysis technology seems particularly important.

$$
\begin{aligned}
& U(x)=(I-C)^{-1} \times C^{T}-\sum_{i=1}^{n} p(x) \times C(i) \sum_{i=1}^{n} p(x) \times Z(i), \\
& V(x)=\frac{\sum_{i=1}^{n} \sum_{j=1}^{n} w(i, j) \times(x(i)-x) \times(x(j)-x)}{\sum_{i=1}^{n} \sum_{j=1}^{n} w(i, j) \times x(i, j)} .
\end{aligned}
$$

Big data have the characteristics of variability, authenticity, and high value. Variability refers to the fact that data are prone to change during the recording process of big data, which increases the difficulty of information processing, and 


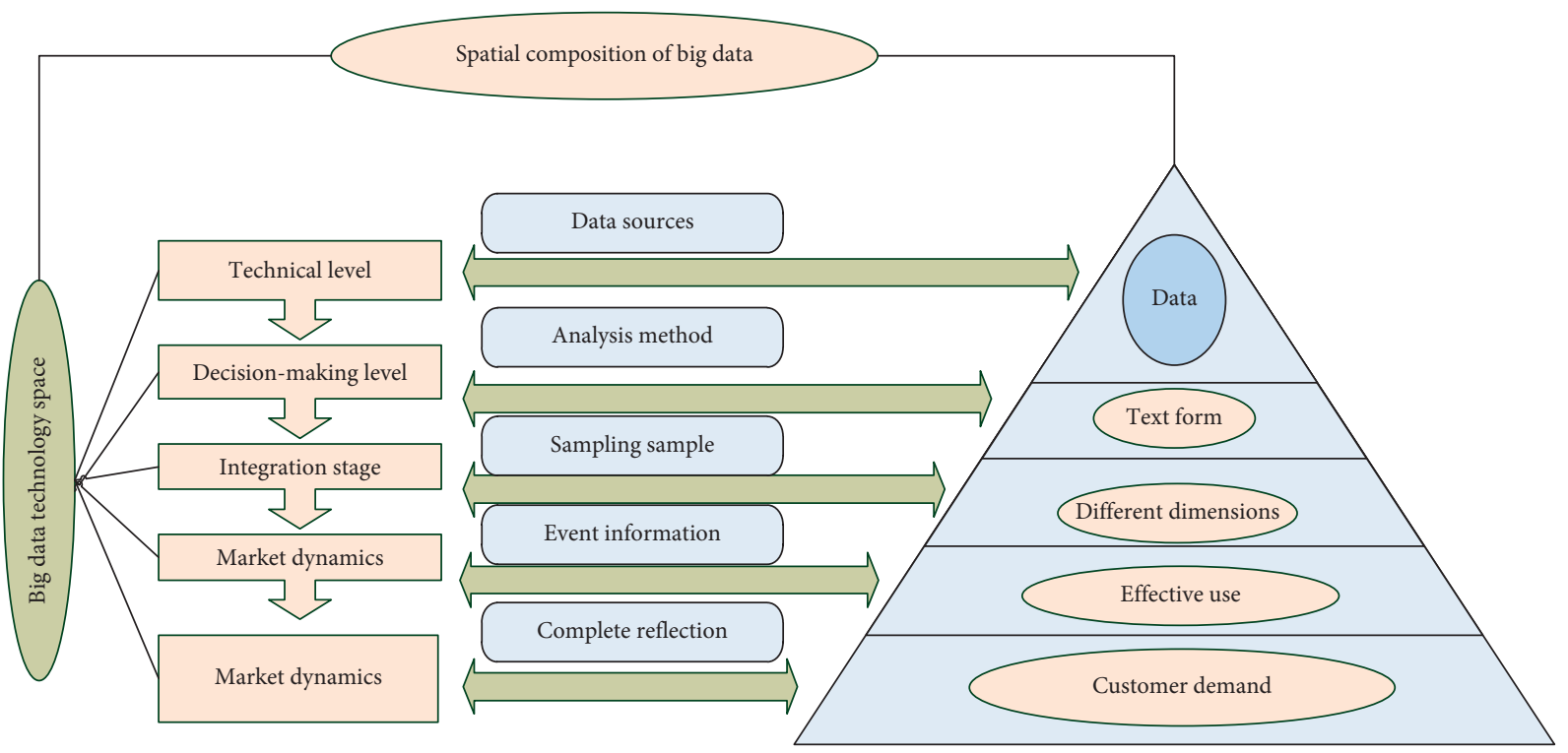

Figure 1: Big data technology space architecture.

complexity is an extension of low density. High value is the most fundamental feature of big data. Its main connotation is that if big data are used reasonably, it can derive greater value. However, the value of big data are directly proportional to the overall amount of big data collected.

\subsection{Interactive Marketing Content Elements. Interactive} marketing technology is a deeper method in big data analysis methods, which mainly refers to the effective analysis of data by appropriate methods from massive amounts of data. Interactive marketing technology provides technical support for precision marketing, and many scholars have made outstanding contributions in this regard. The core of interactive marketing is to analyze the characteristics of consumer behavior and to manage and research consumer groups with common characteristics based on the characteristics of consumer behavior obtained by interactive marketing. The first process of big data analysis is collection. Big data collection refers to the use of big data collection information collection platforms to collect users or other data collection tools. In the process of big data collection, the main problem is that the amount of data information is huge, the amount of data collected is high, and there are many data collection points. In the same period, a large amount of data needs to be collected at the same time. Figure 2 shows the statistical distribution of the deviation of interactive marketing data collection. Therefore, in the process of big data collection, it is necessary to establish a fairly large database, and how to further design the reasonable use and distribution of the database.

The second step is import and preprocessing, which mainly refers to the elimination of invalid information, redundant information, and low-value information after the first collection of information. Therefore, it is necessary to effectively filter and analyze the data before processing. Then, we import the obtained preliminary screening information into another large database. This step is mainly to preprocess the big data. The third step is to conduct statistics and analysis. This process is a process of further refined processing of big data. The effective data are analyzed and filtered, and statistical processing is performed, and finally, effective information is obtained. Different from the above process, big data information mining does not have a definite path or statistical analysis method. The division of labor for small- and medium-sized e-commerce companies has been further refined, and the industrialization of commodities, art, operations, inventory management, and delivery has been formally formed, which has also led to the development of models, express delivery, and other industries. It mainly uses a large amount of data collected in a database and uses various algorithms to perform calculations, which results in complex data. As far as possible to get the effect of prediction or to be able to draw other effective conclusions, the statistical analysis and mining process of big data are considered to be the key process of whether data can be transformed into value space and source of value in the process of big data information processing.

3.3. E-Commerce Platform Construction. E-commerce usually refers to the use of Internet technology, based on the browser/server application method, where buyers and sellers conduct various trade activities on the Internet platform in a face-to-face manner to realize consumers' online shopping, online payment, and various business activities and transaction activities and other new business operating models. Figure 3 shows the distribution of e-commerce platform models. The rise of e-commerce has a close relationship with the development of computer network technology, and the development history of e-commerce has a close relationship with the progress of computer network technology. E-commerce includes many models, typically, B2B (Business to Business), B2C (Business to Consumer), C2C (Consumer 


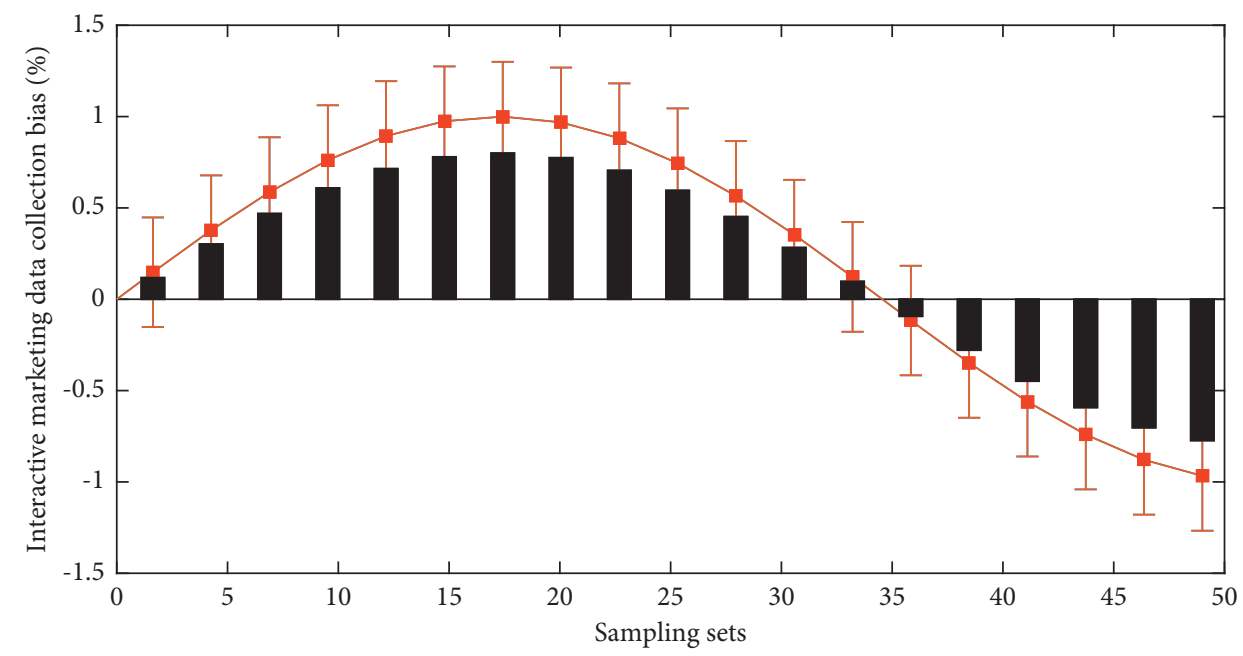

FIgURE 2: Statistical distribution of the deviation of interactive marketing data collection.

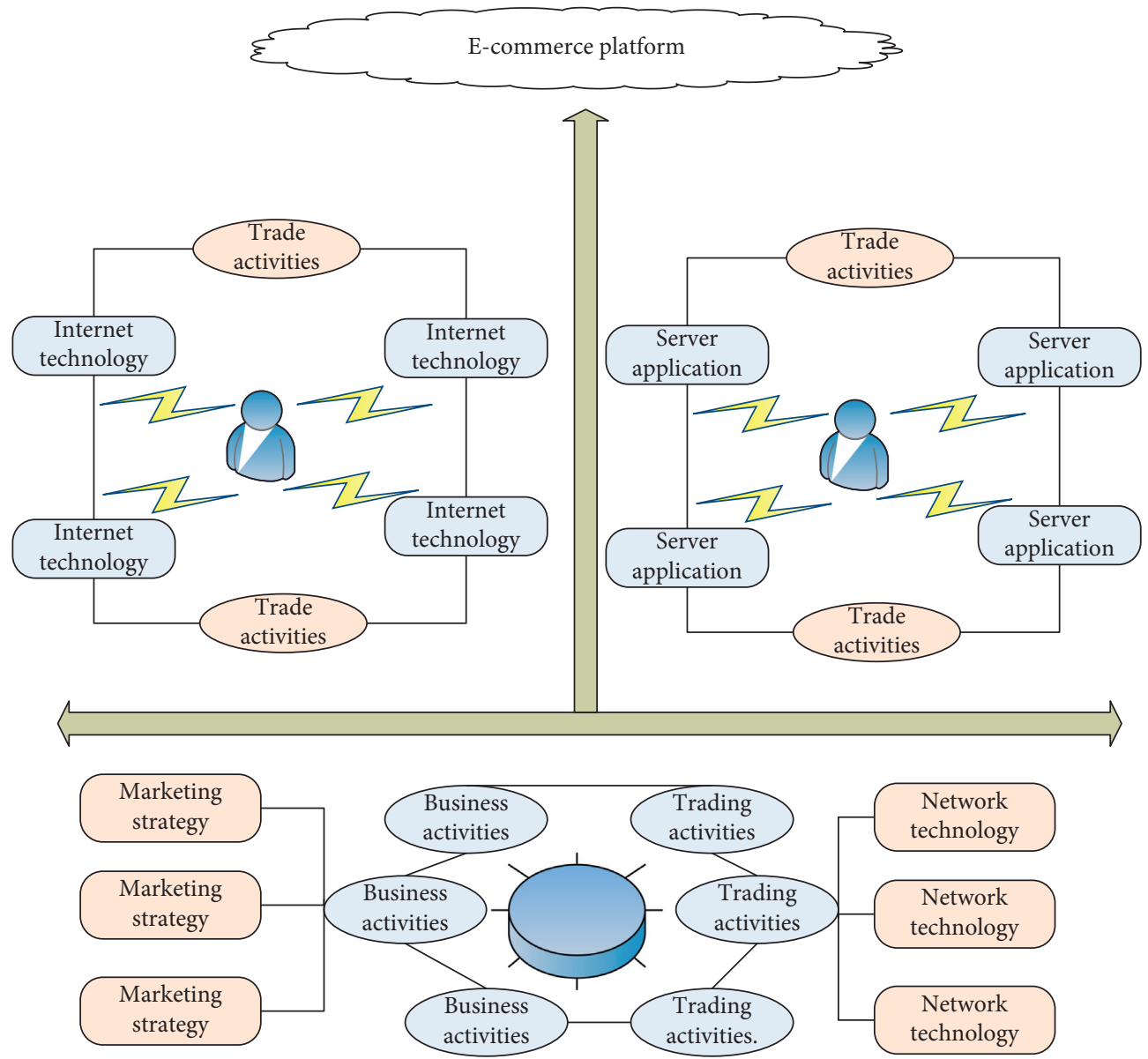

Figure 3: Distribution of e-commerce platform models.

to Consumer), and the recent emergence of P2P (Peer to $\mathrm{Peer}$ ), and $\mathrm{O} 2 \mathrm{O}$ (Online to Offline). Small- and mediumsized e-commerce enterprises refer to small- and mediumsized enterprises that are engaged in e-commerce business (mainly $\mathrm{B} 2 \mathrm{C}, \mathrm{C} 2 \mathrm{C}, \mathrm{O} 2 \mathrm{O}$ ) and conduct e-commerce business on large-scale e-commerce platforms. These enterprises are generally small in scale, with traffic entrances and payment methods. Goods transportation is mainly controlled by large-scale e-commerce platforms and express operators.

E-commerce platform companies provide a good development platform for small- and medium-sized e-commerce companies. Customs clearance is directly attached to 
large-scale e-commerce platforms, saving small- and medium-sized e-commerce companies the trouble of building their own traffic portals. Users can complete what they need on an e-commerce platform. For most of the transactions, the integrated platform operation also helps small- and medium-sized e-commerce companies develop better. However, with the increasingly fierce competition in the industry, the emergence of big data, and the transformation of small- and medium-sized e-commerce companies themselves, market competition has turned to competition characterized by the discovery and satisfaction of user needs, precision marketing strategies, and differentiated competition strategies; the combination of big data strategies is an important path for the continued development of small- and medium-sized e-commerce companies in the future. Precision marketing is a company that pays more attention to marketing actions and results when formulating marketing communication plans. This requires marketing communication and the implementation of marketing activities to be more precise, measurable, and able to achieve higher returns. Small- and medium-sized e-commerce companies have large and small data, simple and relatively complex data, and generally use Access databases or SQL-server databases. Considering that the data types and specific characteristics of user profile data may be more cumbersome, the use of NoSQL databases may be more reasonable and common. NoSQL databases are integrated with the data analysis package, which can efficiently process, organize, and manage data, which has very important advantages. The use of the database is very wide, so it is also widely used by e-commerce companies, with lower usage and maintenance costs.

3.4. System Hierarchical Weight Distribution. After having the database, the next step is to analyze the data. The analysis of the data mainly starts from the two steps of statistical data and exploratory analysis. The statistical data are mainly to analyze the existing data, from which some conclusions can be drawn, such as the popularity of the product, the consumer's preference for buying the product, the relationship between the consumer's demographic characteristics, and the purchase of a certain product or service to compare product sales with similar products or products of different categories in other stores. Table 1 shows the cluster analysis of consumer purchasing preferences. Exploratory analysis is a further level of data analysis. It is mainly to dig out some of the content and conclusions of the existing data that are not represented by the appearance, such as clustering and classification. These require unique analysis techniques, and how big the information can be extent to which the value is played also differs in this process.

The goal of precision marketing is to achieve a low-cost sales strategy through precise customer communication channel selection and sales channel selection, so that both customers and manufacturers can benefit. The core of the word "precision" in precision marketing is to focus on the needs of consumers, which means that the precision marketing system can distinguish a consumer from other
TABLE 1: Cluster analysis of consumer purchasing preferences.

\begin{tabular}{lccc}
\hline $\begin{array}{l}\text { Cluster } \\
\text { index }\end{array}$ & Popularity & $\begin{array}{c}\text { Fusion rate/ } \\
\%\end{array}$ & $\begin{array}{c}\text { Economic efficiency } \\
\text { ratio }\end{array}$ \\
\hline 1 & 0.71 & 82.5 & 1.01 \\
2 & 0.84 & 81.7 & 1.17 \\
3 & 0.69 & 79.6 & 1.14 \\
4 & 0.82 & 77.1 & 1.07 \\
\hline
\end{tabular}

consumers, and recommend the best for consumers according to their needs. It is possible that a certain product or service, instead of the previous emphasis on marketing coverage, should be broad and comprehensive. With the development of information technology, after effective integration, analysis and processing of big data generated by consumers, target consumers can be found among consumer groups, so as to achieve accurate information transmission.

Figure 4 shows the histogram of the economic efficiency of marketing activities. We screen out the information used for identification on the website, such as baby ID; through comprehensive comparison, screen out cross-cutting and interfering data, such as "sales" in the number of sales and successful transactions; do not consider those that do not affect the decision-making factors such as store name, store reputation, and product rating. Specifically, precision marketing means that companies pay more attention to marketing actions and results when formulating marketing communication plans. This requires marketing communications and marketing activities to be implemented more accurately, measurably, and with high returns. In addition, more and more investment in direct sales. In the end, factors that have an impact on the selection of products are left: product specifications, transaction prices, and other customer-related factors; store-related factors such as the number of successful transactions and service commitments; and factors related to platform and regional sales, such as shelves. Precision marketing greatly reduces the cost of investing in unrealistic consumers by discovering potential consumers in consumer groups, improves the economic efficiency of marketing activities, and greatly reduces the cost of marketing activities themselves, but the cost reduction will not weaken the marketing activities themselves, it will increase the accuracy of the activities.

\section{Application and Analysis of Interactive Marketing E-Commerce Recommendation System Driven by Big Data Technology}

4.1. Big Data Feature Extraction. The dependent variable in this study is marketing effect, which can be defined as feedback from media content. This definition mainly measures the short-term effects of self-media marketing. The marketing effect index is expressed by the sum of the number of likes and the number of comments. Typical sampling is used for the selection of self-media platforms. Figure 5 shows the pie chart of the percentage of marketing effect indicators. Sina is selected as the representative from the relevant Weibo platforms, so the data come from Sina Weibo. Its 


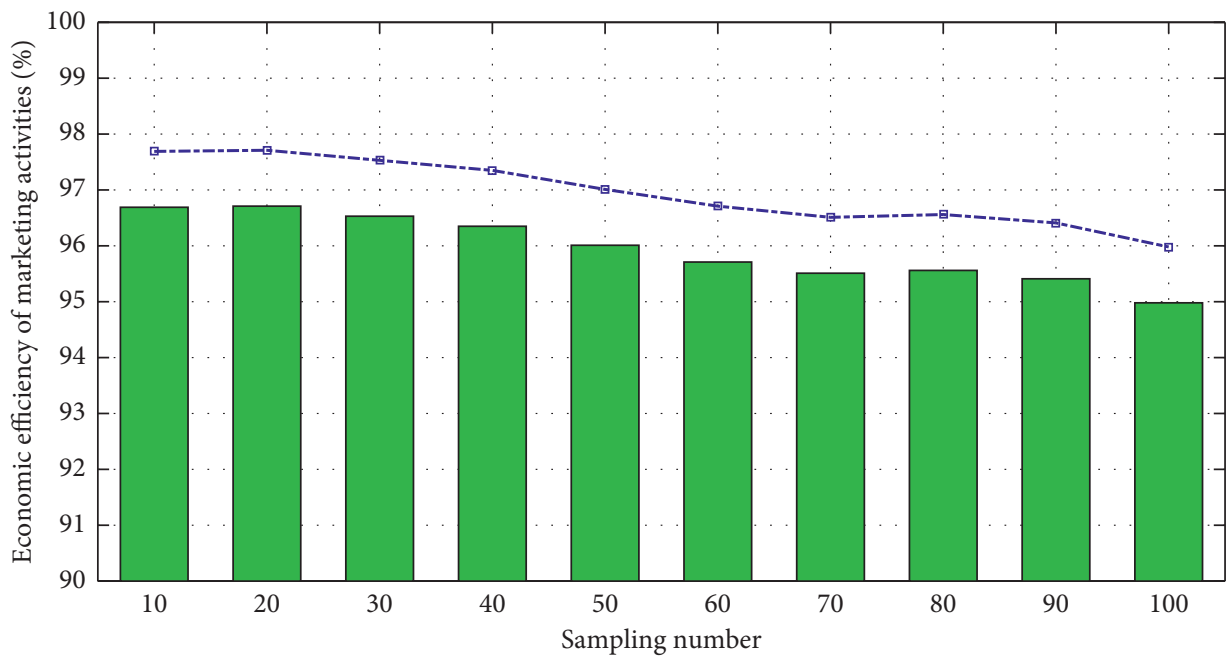

FIGURE 4: Histogram of economic efficiency of marketing activities.
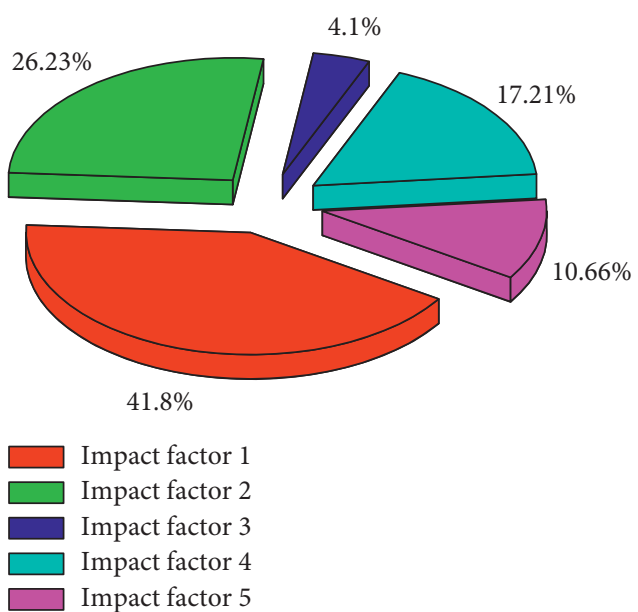

FIGURE 5: Fan chart of the proportion of marketing effect indicators.

representative significance is that Sina Weibo is the largest self-media platform, covering a large consumer group. At the same time, all major interactive marketing e-commerce companies have opened Weibo accounts on Sina Weibo, which is an important platform for interactive marketing e-commerce online marketing, which facilitates horizontal comparison between manufacturers. At the same time, Sina Weibo has the characteristics of open access, can obtain historical information and data, and can provide a sufficient number of samples. Therefore, this article chooses to collect Sina Weibo sample data from a specific period for research.

Using the network data collector, we will analyze the collected 927,174 sets of 268.88 million data. Because these data have the characteristics of large data volume, various types, and low-value density, we will do the data before analysis. After a certain amount of preprocessing, the results of this preprocessing are used to position the target market in terms of channels and regions, and in terms of product attributes and brands, to position consumer demand preferences. We take the seller's ten service promises as the product attribute influencing factors that influence customers to choose between different brands, set it as a dummy variable to introduce the model, and use the MNL model to predict the probability of customers choosing between different brands, which is then the company's selection of promotion channels during the product launch process that provides a basis for decision-making. From the perspective of consumer preferences, we analyze different platform structures through data, and make a certain analysis of which category and which brand of products sell well and the positioning of average prices. Specific to the price range, we discover the potential purchasing power and consumption range of the target customer group; through the analysis of product attributes, we can find out which features of the product will be particularly favored by consumers. Finally, it provides a basis for company A's precise marketing decision on the product.

4.2. E-Commerce System Evaluation. Because the total number of microblogs in the three sample companies exceeded 2,000 within a year, the existing research methods are not sufficient to analyze all of them. Therefore, this article adopts the method of random sampling by computer. We establish a personnel service feedback and evaluation system and establish a personnel efficiency database based on big data. Based on consumer feedback to various service personnel, company internal evaluation system information, and basic information data, the performance of employees can be analyzed and compared, various personnel arrangements can be made, and avoidable accidents can be predicted. The specific steps are to use the random sampling function of the $R$ language, and each manufacturer will sample three months. Secondly, we extract the page numbers of each manufacturer in each month. According to power analysis, this number of microblogs can represent the total sample during the study at a significance level of 0.95. After we have a detailed understanding of the requirements, we need to sort out the data collected on this page. In the end, I chose the store name, product name, price, and number of successful transactions as the collection object. Figure 6 


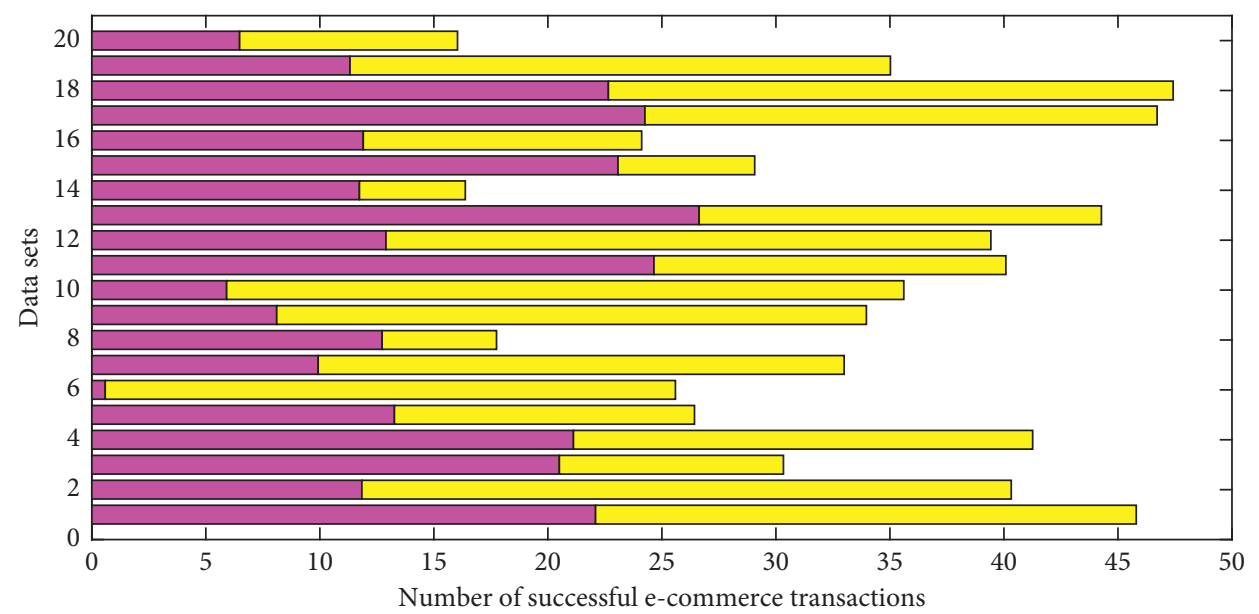

Sample 1

Sample 2

FIgURE 6: Histogram of the number of successful e-commerce transactions.

shows the histogram of the number of successful e-commerce transactions. After completing the data collection, the second step is to classify and filter the data: which are the information used by the website for identification and do not have the value of data exploration and mining; which data are almost duplicated and can be eliminated in half; and which data need to simultaneously consider and get useful data.

The information included in the transaction data that I finally sorted out includes product type, platform, store name, store reputation, product rating, store location, title, baby ID, brand, product specifications, whether it is free shipping, title, price, transaction price, sales volume, number of successful transactions, number of comments, number of favorites, and payment methods. After screening, there are 174 groups of valid and complete data information. Although the target of collaborative filtering technology is specific users, it cleverly uses the information of all users. Recommendations are mainly based on the preferences of other users who are most similar to the user. There are many ways to calculate similarity, such as cosine similarity (the denominator is the square root of the product of the number of products purchased by two users). After we calculate the similarity, we can sort the preferences of other users on the products according to the similarity weighted summation method and then recommend them to specific target users. This collaborative filtering recommendation technology can not only accurately infer the potential needs of users, but more importantly, it can increase cross-sales, that is, when users purchase a certain category of products, they recommend other categories of products to users, thereby increasing the diversity of high user purchases.

4.3. Example Application and Analysis. Content-based recommendation technology is one of the important contents of personalized marketing in the era of big data. This technology originated from information retrieval and information filtering, and its root lies in the acquisition and quantitative analysis of content. Because the research in text information acquisition and filtering is relatively mature, most of the current content-based recommendation systems are mostly the text of the product information operation after analyzing. Coding is to place the analysis unit in the category system. For the first time, we have arranged a week to train coders and improve the coding plan based on the training situation to make the understanding between coders similar to achieve the predetermined reliability. Second, we conduct preresearch to check the reliability of coders and ensure that they master coding skills and methods. Third, in the formal study, standardized tables are used, and computer-aided coding and statistical work are used. Based on similar research, we use a category system to classify each Weibo. The classification is carried out from the three levels of Weibo's basic information, content category, and form, and 21 subcategories are determined to try to exhaust the possibilities of interactive marketing e-commerce microblogs and produce meaningful results.

Figure 7 shows a stick chart of the significance test of e-commerce product categories. To analyze the data of the target product, it is first necessary to separate the data that meet the requirements from the 100 sets of data that have been preliminarily sorted out. After preliminary screening, 100 sets of target product data were obtained, and then, service commitment attributes were selected among them as the data basis for modeling. Next, we need to analyze and process this part of the data. We will use $70 \%$ (the first 50 groups) of samples as training samples for modeling and analysis. In addition to taking security measures to protect consumers' personal information, the industry has also developed a star-rated label on the web page to allow consumers to identify the company's logo for safeguarding consumer data. We will substitute variables into the MNL model and use SAS to test them for empirical analysis, and based on the results of this analysis, we make the choice of promotion channels for the products on the shelves. After 


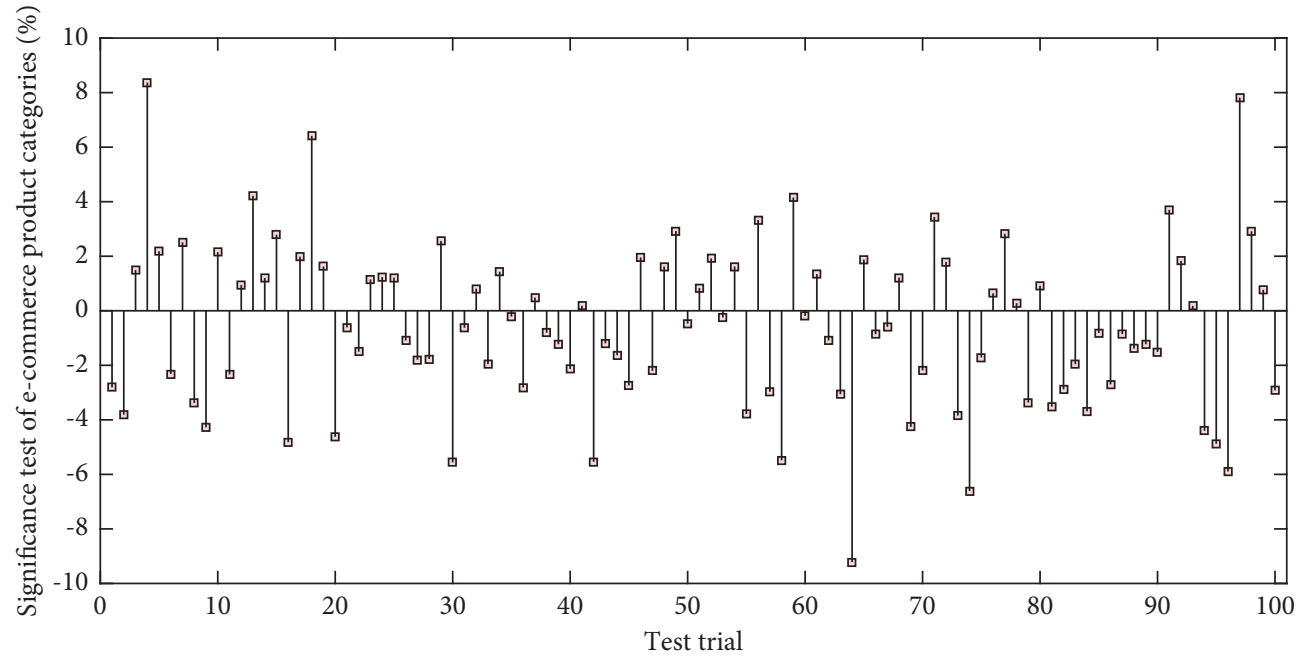

FIGURE 7: Matchsticks for the significance test of e-commerce product categories.

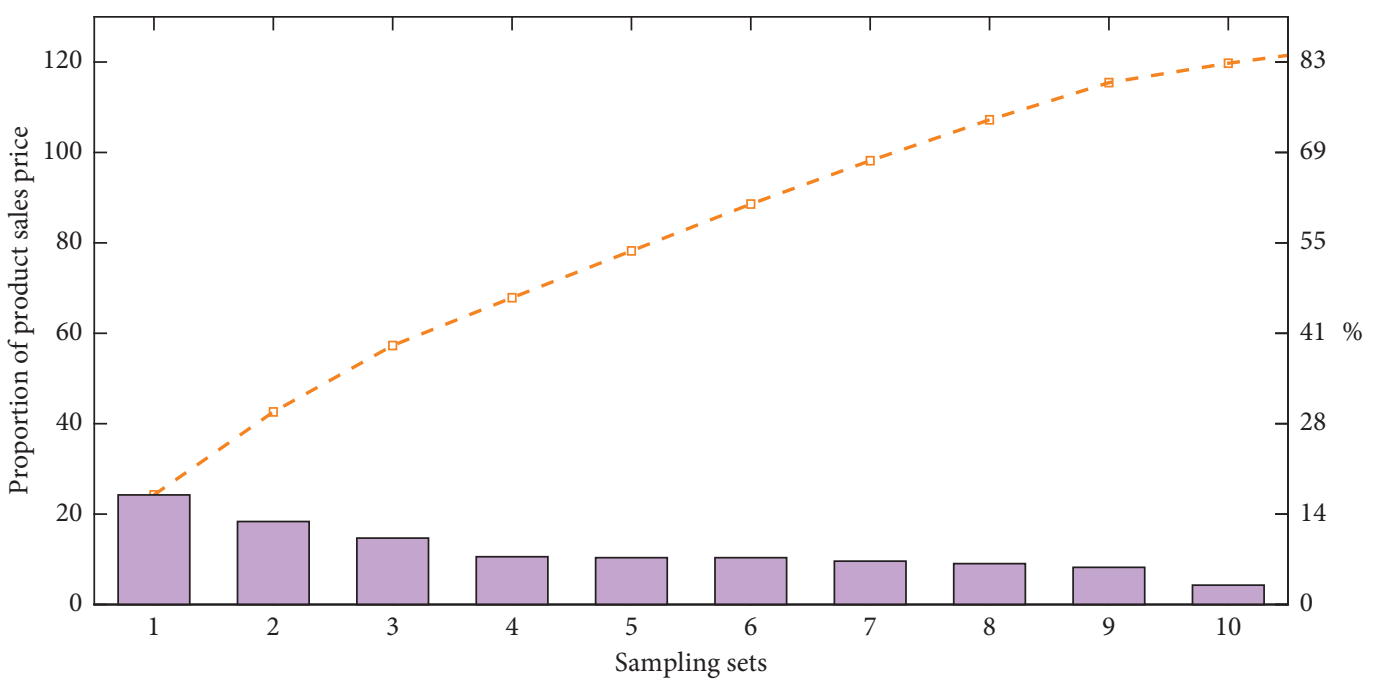

Figure 8: Line chart of product sales price percentage.

the model is obtained, the model is used to predict the remaining 30\% (15 groups) of the test samples, and finally, the prediction results are tested. Using 70\% (the first 50 groups) of samples as training samples, the following results are obtained through repeated screening of variables. Obtaining the data summary, the significance test of the respective variables' influence on the overall model, and the estimated value and significance test of the respective variables are given. It can be seen that the variables VAR18, VAR19, and VAR25 all passed the significance test. The last line is the likelihood ratio test of the goodness of fit of the model. The likelihood ratio statistic is 6.93 , and the statistical result is not significant $(p=0.0743)$, which shows that the fitted model is acceptable. The last column gives the results of the significance test of each independent variable for each MNL model, in which the coefficient estimates of the remaining independent variables are not significant except for the estimated value of the second coefficient of VAR18 and the estimated value of the third coefficient of VAR19. All passed the significance test.

Figure 8 shows a line chart of the percentage of product sales prices. It can be seen that in terms of product prices, from the perspective of Taobao's entire network, the product industry's most shipped price range is between 11 yuan and 22 yuan, of which shipments accounted for $27.9 \%$, and the high sales price range is between 22 yuan and 33 yuan, of which sales accounted for $14.3 \%$. In addition, there is a significant increase in the range of 333-432 yuan, indicating that most consumers still choose relatively low-end cosmetics and do not pursue branded products; the other part tends to buy branded products, focusing on products purchased in several well-known brands, although the products at the midprice range are seldom bought. Judging from the basic information of the research subjects, they have the ability to read and understand the questionnaire of this research and can accurately express their opinions. 
Therefore, the data of this questionnaire are valid. The sales of normal specifications accounted for the highest proportion, accounting for nearly $90 \%$ of the market share, whereas the choice of suits was less, indicating that consumers' demand is only to choose a certain type or a certain fragrance of the product according to their preferences, rather than buying a certain brand. For a full set of products, some consumers tend to prefer to purchase the corresponding light-backed products, and such consumers may prefer to use them for their own use.

\section{Conclusion}

This article mainly studies the precision marketing system based on big data, sorts out the history of e-commerce development, discusses the possibility of combining big data and precision marketing systems, and analyzes the major e-commerce platforms and enterprise application of big data technology in the era of big data. At the same time, the study is carried out with it as a typical case, focusing on small and medium e-commerce companies, especially small e-commerce companies that rely on e-commerce platforms, such as Amazon, JD.com, and Taobao, to carry out the real possibility and necessity of precision marketing based on big data. Based on practical factors, this article designs a general system platform based on general big data precision marketing strategies and uses clustering algorithms as an example to illustrate the use of the system. At the same time, from the aspects of improving enterprises' understanding of big data strategy and precision marketing, applying big data to adjust corporate marketing strategies, precision marketing channels, deepening consumer demand analysis, applying big data analysis results to optimize business operations, and the like, it proposes to optimize the precision marketing of system countermeasures and suggestions. In most cases, the measurement of validity is the measurement of construction validity, and the measurement of construction validity is carried out through confirmatory factor analysis. Specifically, it is judged by the standardized factor loading of the item, if the fit is good, then it can be further tested for validity through standardized factor loading coefficients. This study avoids the complicated model construction and high-specialization design and selects the MNL model (Multinominal Logit Model), which is simple in model construction and widely used in data analysis, to quantitatively construct the predictive model and obtain the customer's purchase probability, and it achieved a high accuracy rate, rather than a general qualitative analysis, so that small and micro e-commerce merchants can more targeted product selection and sales, which can provide a basis for e-commerce precision marketing but also have a high actual operation. We hope that through the analysis of typical cases in this article, we can develop precision marketing strategies for small- and medium-sized e-commerce companies in the future and provide a good reference and experience.

\section{Data Availability}

The data used to support the findings of this study are included within the article.

\section{Conflicts of Interest}

The authors do not have any possible conflicts of interest.

\section{References}

[1] Y. Liu, J. Lu, F. Mao, and K. Tong, "The product quality risk assessment of e-commerce by machine learning algorithm on spark in big data environment," Journal of Intelligent and Fuzzy Systems, vol. 37, no. 4, pp. 4705-4715, 2019.

[2] F. Pinarbasi and Z. N. Canbolat, "Big data in marketing literature: a Bibliometric Analysis," International Journal of Business Ecosystem \& Strategy, vol. 1, no. 2, pp. 15-24, 2019.

[3] Q. Guo, C. Yang, and S. Tian, "Prediction of purchase intention among E-commerce platform users based on big data analysis," Revue d'Intelligence Artificielle, vol. 34, no. 1, pp. 95-100, 2020.

[4] E. Taiebi Javid, M. Nazari, and M. R. Ghaeli, "Social media and e-commerce: a scientometrics analysis," International Journal of Data and Network Science, vol. 3, no. 3, pp. 269-290, 2019.

[5] H. Xu, K. Li, and G. Fan, "Novel model of e-commerce marketing based on big data analysis and processing," in Proceedings of the 2017 International Conference on Computer Network, Electronic and Automation (ICCNEA), pp. 80-84, Computer Network, Electronic and Automation, Xi'an, China, September 2017.

[6] A. Pradana, G. O. Sing, and Y. J. Kumar, "SamBot-intelligent conversational bot for interactive marketing with consumercentric approach," International Journal of Computer Information Systems and Industrial Management Applications, vol. 6, no. 2014, pp. 265-275, 2017.

[7] A. Behl, P. Dutta, S. Lessmann, Y. K. Dwivedi, and S. Kar, “A conceptual framework for the adoption of big data analytics by e-commerce startups: a case-based approach," Information Systems and E-Business Management, vol. 17, no. 2, pp. 285-318, 2019.

[8] Y. Jiang, X. Gu, D. Wu et al., "A novel negative-transferresistant fuzzy clustering model with a shared cross-domain transfer latent space and its application to brain CT image segmentation," IEEE/ACM Transactions on Computational Biology and Bioinformatics, vol. 18, no. 1, pp. 40-52, 2021.

[9] Y. Jiang, Y. Zhang, C. Lin, D. Wu, and C.-T. Lin, "EEG-based driver drowsiness estimation using an online multi-view and transfer TSK fuzzy system," IEEE Transactions on Intelligent Transportation Systems, vol. 22, no. 3, pp. 1752-1764, 2021.

[10] P. A. Hurtado, C. Dorneles, and E. Frazzon, "Big Data application for E-commerce's Logistics: a research assessment and conceptual model," IFAC-PapersOnLine, vol. 52, no. 13, pp. 838-843, 2019.

[11] M. Chen and Q. Du, "E-commerce marketing strategy based on big data statistical analysis," in Proceedings of the 13th International Conference on Measuring Technology and Mechatronics Automation (ICMTMA), pp. 686-689, Measuring Technology and Mechatronics Automation, Beihai, China, January 2021.

[12] L. Li and J. Zhang, "Research and analysis of an enterprise E-commerce marketing system under the big data environment," Journal of Organizational and End User Computing, vol. 33, no. 6, pp. 12-19, 2021.

[13] B. Li, "Analysis of the impact of E-commerce on industrial manufacturing based on big data," Journal of Physics: Conference Series, vol. 1648, no. 4, Article ID 042106, 2020.

[14] L. Liang and Y. H. Pan, "Research on user experience under the new retailing mode: using the interactive marketing mode 
of the estee lauder POP-UP store as an example," Journal of Digital Convergence, vol. 19, no. 6, pp. 343-353, 2021.

[15] L. Shang, "Research on using big data marketing to promote the development of E-commerce under information management mode," Journal of Physics: Conference Series, vol. 1792, no. 1, Article ID 012026, 2021.

[16] D. Malhotra and O. Rishi, "An intelligent approach to design of E-Commerce metasearch and ranking system using nextgeneration big data analytics," Journal of King Saud University-Computer and Information Sciences, vol. 33, no. 2, pp. 183-194, 2021.

[17] J. Hu, "E-commerce big data computing platform system based on distributed computing logistics information," Cluster Computing, vol. 22, no. 6, pp. 13693-13702, 2019.

[18] L. T. Wright, R. Robin, M. Stone, and E. Aravopoulou, "Adoption of Big Data technology for innovation in B2B marketing," Journal of Business-To-Business Marketing, vol. 26, no. 3-4, pp. 281-293, 2019.

[19] G. Elia, G. Polimeno, G. Solazzo, and G. Passiante, "A multidimension framework for value creation through big data," Industrial Marketing Management, vol. 90, pp. 617-632, 2020.

[20] C. Liu, S. Wang, and G. Jia, "Exploring E-commerce big data and customer-perceived value: an empirical study on Chinese online customers," Sustainability, vol. 12, no. 20, Article ID $8649,2020$.

[21] B. Zhang, Z. Du, B. Wang, and Z. Wang, "Motivation and challenges for e-commerce in e-waste recycling under "big data" context: a perspective from household willingness in China," Technological Forecasting and Social Change, vol. 144, pp. 436-444, 2019.

[22] M. Yang and S. Hu, "Research on the E-business platform of agricultural products and rice marketing channel based on network big data," Revista Técnica de la Facultad de Ingeniería Universidad del Zulia, vol. 39, no. 12, pp. 258-265, 2016.

[23] Y. Kim and R. A. Peterson, "A meta-analysis of online trust relationships in E-commerce," Journal of Interactive Marketing, vol. 38, pp. 44-54, 2017. 